\title{
Opioid-Free Naloxone Emergence Under Dexmedetomidine: A Case Report
}

\author{
Robert J. Long ${ }^{1}$, Henry R. DeYoung ${ }^{1}$, Gregory J. Booth ${ }^{1}$, Marcus G. Herod ${ }^{1}$
}

\section{Abstract}

This report describes a case in which naloxone appeared to expedite emergence from general anesthesia in a patient who underwent an opioid-free anesthetic that included dexmedetomidine. After administration of naloxone, the patient progressed rapidly from general anesthesia to alert and oriented in approximately two minutes. Additionally, the patient demonstrated rapid reversal of miosis. He also reported adequate analgesia in the PACU, requiring no opioids and denied any recall of events that occurred prior to the administration of naloxone.

Keywords: Naloxone; Dexmedetomidine; Delayed emergence.

Introduction

Dexmedetomidine is a highly selective and potent $\alpha 2$ adrenoreceptor (alpha-2R) agonist. It decreases sympathetic outflow in the central nervous system (CNS), providing opioid-sparing analgesic and amnestic properties free of respiratory depression. Common side effects include hypotension, bradycardia, and sedation. These effects primarily occur through hyperpolarization of the noradrenergic neurons in the locus coeruleus of the brain stem, the primary site modulating wakefulness. This mechanism also leads to decreased nociceptive transmission, similar to opioid receptor activation [1]. Alpha-2R and opioid receptor agonists are thought to have synergistic effects by modulating different receptors working through a common pathway [2]. Further, alpha-2R-agonists have been shown to induce analgesia via increased release of endogenous opioids [3].

Naloxone is a competitive opioid receptor antagonist at the $\mathrm{mu}$, kappa, and delta receptors with a rapid onset of action. It has a well-established role in the reversal of opioid receptor agonists $[4,5]$. There are several reports of administering naloxone to reverse the effects of other alpha-2R-agonists, but none that discuss the use of naloxone to reverse the sedative effects of dexmedetomidine in humans [7-13].

In this case report, we believe naloxone may have contributed to an expedited emergence from general anesthesia, which was maintained, in part, by dexmedetomidine.

\section{Case Report}

A 45-year-old male underwent a planned C5-C6 Anterior Cervical Fusion and Discectomy under general endotracheal intubation anesthesia (GETA), utilizing an opioid free anesthetic technique. Preoperatively, he received intravenous (IV) midazolam $2 \mathrm{mg}$ and was induced with propofol $200 \mathrm{mg}$, lidocaine $100 \mathrm{mg}$, and ketamine $20 \mathrm{mg}$, and was maintained under sevoflurane. Following induction, he received dexmedetomidine $200 \mathrm{mcg}$ intranasally, which was chosen per provider preference. Total anesthesia time until surgery completion was 120 minutes, followed by delayed emergence lasting approximately 50 minutes. During this period, the patient maintained a strong respiratory drive and had an end-tidal sevoflurane concentration of $<0.1 \%$. A thorough physical exam was performed, notable for miosis. Arterial blood gas was unremarkable. Out of concern for possible unknown opioid administration and prior to further work up for an unanticipated neurologic complication, the patient was given naloxone $40 \mathrm{mcg}$. Within two minutes, he displayed a sudden and dramatic emergence with resolution of miosis. He reported no memory of the events prior to emergence and no significant pain. A urine drug screen was negative for opioids. The patient denied taking any opioids himself prior the surgery and with his particular military position he is tested with urine drug screen regularly. No opioids were removed for the case and all other opioids were accounted for in other cases that day.

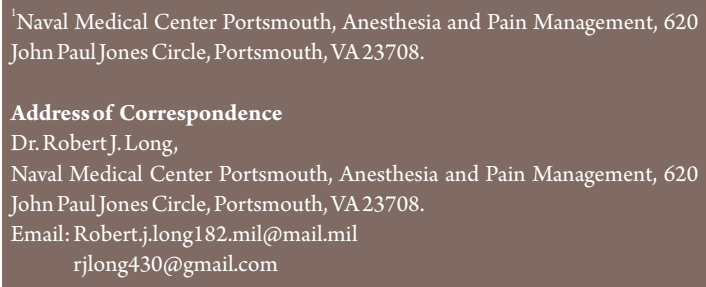

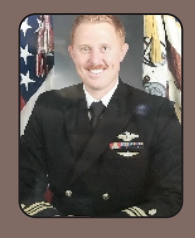

Dr. Robert J. Long
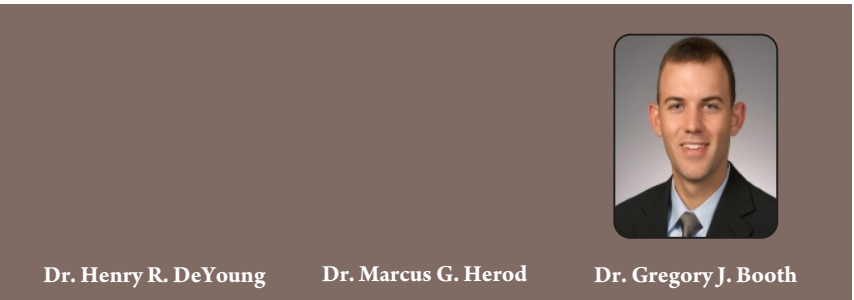

2021 (C) Journal of Anaesthesia and Critical Care Case Reports| Available on www.jaccr.com | ISSN 2454-7174 | DOI: 10.13107/jaccr.2021.v07i01.167 This is an Open Access article distributed under the terms of the Creative Commons Attribution Non-Commercial License (http://creativecommons.org/licenses/by-nc/3.0) which permits unrestricted non-commercial use, distribution, and reproduction in any medium, provided the original work is properly cited. 


\section{Discussion}

Administration of naloxone in this case was temporally related to dramatic emergence from general anesthesia. Extensive evaluation for the etiology of delayed emergence was unrevealing.

Delayed emergence is defined as the failure to recover consciousness 30-60 minutes after general anesthesia, and the clinical presentation varies. The scarcity of clinical studies on this phenomenon has limited the discovery of its true incidence and made it difficult to comprehend the complexity of this postoperative complication. The most common causes of delayed emergence are residual anesthetic agents, drug interactions, polypharmacy, surgical complications, neurologic sequelae, endocrine/metabolic derangement, and psychiatric conditions. Some other uncommon reasons include pseudocholinesterase deficiency, serotonin syndrome, central anticholinergic syndrome, and narcolepsy/sleep paralysis [16]. In this case the patient's vital signs, body temperature, $A B G$ results, glucose level, and $\mathrm{ETCO}_{2}$ were within normal limits. Twitch monitor, volatile gas monitor and EKG strip also showed unremarkable results that could not explain the continued general anesthesia level of sedation. As the case involved the cervical spine, there was also concern for possible surgical neurologic complications and CT studies were being considered. After the physical exam revealed miosis, the providers elected to administer naloxone to address the potential for inadvertent or unknown opioid administration and possibly to avoid exposing the patient to and an unnecessary radiation. Given the lack of reported history of opioid use, negative urine drug screen, and accurate accounting of all opioids used in operating suite that day, the authors feel it is exceedingly unlikely that the dramatic emergence was due to reversing the effects of opioids.

Dexmedetomidine-induced miosis, thought to result from a central sympatholytic effect mediated by the cerulean-spinal pathway and associated with hypotension and bradycardia has been described [7]. This evidence, in the context of the case described, spurred interest that naloxone may also reverse sedation from dexmedetomidine. With the rapid and full emergence following naloxone admiration in an opioid free patient, the etiology of delayed emergence from other common and uncommon causes was deemed highly unlikely. After an extensive literature review, there were no reports of naloxone's effects on emergence from non-opioid anesthesia or human studies describing its reversal of dexmedetomidine. However, human studies describing naloxone interaction and reversal of similar $\alpha 2$-adrenoceptor drugs including clonidine, guanfacine, and more were found [7-13].

Several reports describe using naloxone to treat hemodynamic and sedative effects caused by clonidine [713]. One report described reversal of sedation in 40 out of 51 pediatric patients with clonidine exposure, albeit at doses of naloxone up to $10 \mathrm{mg}$ [11]. Conversely, clonidine was shown to abolish the muscle sympathetic activity induced in opioidaddicted patients following naloxone administration, supporting the notion of an antagonistic relationship between clonidine and naloxone [7].

The clonidine-naloxone interaction may stem from endogenous opioids that are released or modulated by clonidine. One report found a significant correlation between the clonidine-induced increase in plasma betaendorphin level and the naloxone-induced change in mean blood pressure [13]. Additionally, the interaction may represent a combination of effects on endogenous opioids, sympatholytic actions, different opioid receptors, central vs peripheral receptors, and Alpha- $2 a, 2 b$, and $2 c$ receptor subtypes $[1,7-13]$. Naloxone's antagonizing effect on alpha$2 \mathrm{R}$ agonists extends beyond clonidine. A case report describes naloxone reversal of sedation, hypotension, and bradycardia caused by guanfacine [11]. Additionally, an animal model revealed that reward behavior induced by dexmedetomidine is reversed by naloxone, suggesting a putative opioid-mediated effect of dexmedetomidine [14]. There are many studies describing various physiologic effects of endogenous opioids, but none that describe their effects on level of consciousness.

Reports are conflicting, however, with one literature review concluding that naloxone should not be given empirically for clonidine intoxication [10]. The evidence is difficult to interpret as many cases involve intoxication with unknown amounts of clonidine, such as a young child found with an open pill bottle. It is unclear if the variable success of naloxone to reverse clonidine is due to lack of efficacy, unclear dose-response relationship, or some other effect such as patient comorbidities or genetic variability.

Naloxone has many reported side effects such as cardiac dysrhythmias, pulmonary edema, and seizures that should be considered prior to administration in any patient. Many of the sides effects of naloxone have been documented following administration of opioids and the resultant sudden reversal or withdraw. At the time of administration in this case, there was concern for unknown opioid administration in a patient with delayed emergence. We could not find any studies describing naloxone side effects profile or its effect on conscious without co-administration of opioids. In this case the administration of low dose naloxone likely prevented the patient from receiving unnecessary radiation and other procedures.

Albeit limited and sometimes contrary, we've presented biochemical and clinical evidence of interactions between 
naloxone and clonidine, clinical evidence of interaction between naloxone and guanfacine, and evidence of naloxone's reversal of dexmedetomidine-induced behavioral effects in an animal model. It follows that there is a plausible antagonistic interaction between dexmedetomidine and naloxone in humans.

However, there are several limitations that must be addressed. Much of the evidence describing naloxone use for clonidine toxicity describes doses which are orders of magnitude larger than the doses we administered. The bioavailability of intranasal dexmedetomidine and the context sensitive half-life of dexmedetomidine infusions are both highly variable. Further, several sedative hypnotic agents were used, so it is impossible to determine to what extent dexmedetomidine specifically, or synergistically with other medications, contributed to sedation.

Ketamine demonstrates mu-opioid agonism and opioid potentiation, so it is possible that naloxone primarily reversed effects of ketamine, although the interval and dose between the $20 \mathrm{mg}$ of ketamine and the naloxone dose administration argues against this [15]. We also cannot rule out coincidental administration of naloxone and emergence from anesthesia which may have been unrelated to naloxone. This is less likely with the delayed emergence time of $50 \mathrm{~min}$, and the rapid emergence from being unresponsive to pain, to awake and alert within 2 min of the naloxone dose. Finally, inadvertent or undetected opioid administration is possible, although unlikely given the lack of reported medical history, maintenance of strong respiratory drive, use of proprietary medication vials, and negative urine drug screen.

Our experience generated a very intriguing hypothesis - can naloxone effect rapid emergence from general anesthetics that use dexmedetomidine and no opioids? When confronted with depressed cognition or delayed emergence, can naloxone be considered in anesthesia or critical care without prior administration of opioids? We have designed a randomized control trial to investigate this question systematically and hope to shed more light on the topic in the near future.

Glossary of Terms: alpha-2R: $\alpha 2$-adrenoreceptor, CNS: Central Nervous System, PACU: Post Anesthesia Care Unit, GETA: General Endotracheal Anesthesia.

Disclaimer: The views expressed in this case are those of the authors and do not necessarily reflect the official policy or position of the Department of the Navy, Department of Defense, or the United States Government.

\section{References}

1. Naaz S, Ozair E. Dexmedetomidine in current anesthesia practice- a review. Journal of clinical and diagnostic research: JCDR. 2014;8(10):Ge01-4.

2. Chabot-Doré AJ, Schuster DJ, Stone LS, Wilcox GL. Analgesic synergy between opioid and a2-adrenoceptors. British journal of pharmacology. 2015;172(2):388-402

3. Sullivan AF, Kalso EA, McQuay HJ, Dickenson AH. The antinociceptive actions of dexmedetomidine on dorsal horn neuronal responses in the anaesthetized rat.Eur J Pharmacol. 1992;215(1):127-33.

4. Boyer EW. Management of opioid analgesic overdose. The New England journal of medicine. 2012;367(2):146-55.

5. Helm S, Trescot AM, Colson J, Sehgal N, Silverman S. Opioid antagonists, partial agonists, and agonists/antagonists: the role of office-based detoxification. Pain physician. 2008;11(2):225-35.

6. Keinbaum, P., et al. Sympathetic Neural Activation Evoked by $\mu$-Receptor Blockade in Patients Addicted to Opioids Is Abolished by Intravenous Clonidine. Anesthesiology. 2002: 96:346-351.

7. Farsang C, Kunos G. Naloxone reverses the antihypertensive effect of clonidine. British journal of pharmacology. 1979;67(2):161-4.

8. Kulig K, Duffy J, RumackBH, Mauro R, Gaylord M. Naloxone for treatment of clonidine overdose. Jama. 1982;247(12):1697.

Conflict of Interest: Nil

Source of Support: None
9. Cook P. Clonidine-induced unconsciousness: reversal with naloxone. Anaesthesia and intensive care. 1987;15(4):470-1.

10. Ahmad SA, ScolnikD, Snehal V, Glatstein M. Use of naloxone for clonidine intoxication in the pediatric age group: case report and review of the literature. Am JTher. 2015 Jan-Feb. 22 (1):e14-6.

11. Tsze DS, Dayan PS. Treatment of guanfacine toxicity with naloxone. Pediatric emergency care. 2012;28(10):1060-1.

12. Seger, D. and Loden, J. Naloxone reversal of clonidine toxicity: Dose, Dose, Dose. Clinical Toxicology.2018:56(10):873-879.

13. Niemann JT, Getzug T, Murphy W. Reversal of clonidine toxicity by naloxone. Annals of emergency medicine. 1986;15(10):1229-31.

14. 12. Uskur T, Barlas MA, Akkan AG, Shahzadi A, Uzbay T. Dexmedetomidine induces conditioned place preference in rats: Involvement of opioid receptors. Behav Brain Res. 2016;296:163-8.

15. Sleigh, J., et al. Ketamine - More mechanisms of action than just NMDA blockade. Trends in Anaesthesia and Critical Care. 2014:4(2-3):76-81.

16. Cascella M, Bimonte S, Di Napoli R. Delayed Emergence from Anesthesia: What We Know and How We Act. Local Reg Anesth. 2020 Nov 5; 13:195206. doi: 10.2147/LRA.S230728.

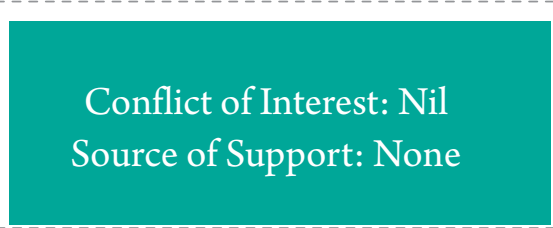

How to Cite this Article

Long RJ, DeYoung HR, Booth GJ, Herod MG | Opioid-Free Naloxone Emergence Under Dexmedetomidine: A Case Report | Journal of Anaesthesia and Critical Care Case Reports | January-April 2021; 7(1): 11-13.

13 | Journal of Anaesthesia and Critical Care Case Reports | Volume 7 | Issue 1 | January-April 2021 | Page 11-13 